\title{
Mitogen-Activated Protein Kinase
}

National Cancer Institute

\section{Source}

National Cancer Institute. Mitogen-Activated Protein Kinase. NCI Thesaurus. Code C16376.

Mitogen-activated protein kinases (MAPKs) are serine-threonine protein kinases activated in response to a variety of extracellular stimuli and encoded by a multigene family. The MAPKs are part of complex protein kinase cascades. One of the several classes of MAPKs includes (among other ERKs) the extracellular signal-regulated kinases ERK1 (p44MAPK) and ERK2 (p42MAPK), activated by the RAF/MEK/ERK signaling pathway. ERK1/2 are widely involved in eukaryotic signal transduction. Upon activation, ERKs translocate to the nucleus and phosphorylate nuclear targets. Phosphorylated ERKs form essential dimers with phosphorylated and unphosphorylated ERK partners.

Dimerization is part of the mechanism of MAP kinase family action. Mitogen-activated protein kinases (MAPKs) promote entry in the cell cycle. (from OMIM 601795, 176948, Swiss-Prot P27361, P28482 and NCI) 